Tilen Thaler, Blaž Krese, Edvard Govekar,

Stability diagrams and chatter avoidance in horizontal band sawing,

CIRP Annals,

Volume 64, Issue 1,

2015,

Pages 81-84,

ISSN 0007-8506.

(C) 2015. This manuscript version is made available under the CC-BY-NC-ND 4.0 license http://creativecommons.org/licenses/by-nc-nd/4.0/

Link to published article:

http://www.sciencedirect.com/science/article/pii/S000785061500089X

DOI: https://doi.org/10.1016/j.cirp.2015.04.081 


\title{
Stability diagrams and chatter avoidance in horizontal band sawing
}

\author{
Tilen Thaler a, Blaž Krese a, Edvard Govekar a (1) \\ University of Ljubljana, Faculty of Mechanical Engineering, Ljubljana, Slovenia
}

The paper presents recurrence plot based stability analysis of the horizontal band sawing process of structural steel profiles. The analysis is performed in the parameter space defined by the cutting speed, the distance between the blade supports, and the feed rate. The corresponding stability diagrams have been constructed using the recurrence plot characteristic, the determinism of the sound pressure emitted by the process, which quantifies the process predictability. The topology of the experimentally obtained stability diagrams revealed non-linear nonmonotonic dynamic behaviour, which made two different chatter avoidance strategies possible by cutting speed variation.

Cutting, Chatter, Band sawing

\section{Introduction}

Instability related chatter phenomena are commonly observed in various machining processes [1]. Consequently instability related phenomena in these processes have received considerable attention since high-amplitude vibrations can cause striations on the cut surface, the so-called 'wash-boarding' effect [2], which means that additional treatment is needed if the final functionality of the product is not to be reduced.

Band sawing is a process which involves the use of a multicutting edge tool and has at least two main adjustable process parameters (cutting speed and feed rate). There are also phenomena of dynamic force excitation, non-linear friction in the cutting zone, and non-linear plastic deformation of the workpiece in the cutting zone, all of which add to the process complexity. Since analytical treatment of the problem of cutting process stability analysis is difficult to adapt to industrial conditions, experimental approaches are very often applied.

Power spectrum characteristics in the frequency domain [3] [4] [5] [6] [7] and the wavelet transform [8] of various sensory signals are most often use for the experimental characterization of stability and related chatter during cutting processes. However, the drawback of using power spectrum or wavelet based characterization is that the informative frequency components depend on the modal parameters of the mechanical system being tested. In order to avoid this, and to give the presented analysis more inherent physical merit, it is proposed that recurrence plots, as first introduced by Eckmann et al. [9], could be used for the construction of stability diagrams in band sawing. Such recurrence plots, together with the corresponding recurrence quantification analysis (RQA), have been shown to be applicable for the characterization of dynamic behaviour in various fields of science and engineering [10] [11]. Notably, recurrence plots have already been used for chatter characterization and detection in turning [12], where it was qualitatively established that chatter is similar to a deterministic process, while chatter-free cutting has the properties of a stochastic process.

In this paper a novel RQA has been employed for the construction of stability diagrams in a band sawing parameter space defined by the cutting speed, the distance between the blade supports, and the feed rate. The method requires estimation of the selected RQA statistic, which is calculated from the fluctuations of the sound pressure emitted by the process. Values of the statistic close to zero are characteristic of chatter free, whereas values close to one are characteristic of chatter band sawing. Finally the adequacy of the constructed stability diagrams was investigated in a chatter avoidance experiment, which showed that chatter avoidance with increasing cutting speed is more appropriate than with decreasing cutting speed.

\section{Experimental setup}

Experiments were conducted on a double column PE-TRA Toolmaster 300DC band saw with a maximum cutting width capacity of $300 \mathrm{~mm}$. A bimetal cutting blade with a length of 4150 $\mathrm{mm}$ and a pitch of 3-4 teeth per inch was used, and tensioned at $2.0 \mathrm{kN}$. Detailed characteristics about the band saw machine and the cutting blade are given in Table 1 .

Table 1 Cutting blade parameters.

\begin{tabular}{ll} 
Parameter & Value \\
\hline Material & M42 \\
Loop length [mm] & 4150 \\
Width/Thickness [mm] & $34 / 1.1$ \\
Teeth pitch [teeth per inch] & $3-4$ \\
Rake/Clearance angle $\left[^{\circ}\right]$ & $10 / 32$ \\
\hline
\end{tabular}

The band saw was equipped with a Brüel \& Kjær microphone in order to measure the sound pressure $p(t)$, and with a Kistler three-component dynamometer in order to measure the cutting force $\boldsymbol{F}(t)$ that were generated during the band sawing process. The positions of the microphone and the dynamometer are shown in Fig. 1. Data about the sound pressure $p(t)$ and the cutting force $\boldsymbol{F}(t)$ were acquired by means of a 16-bit resolution A/D data acquisition card, and transferred into a personal computer for further off-line analysis. The sampling frequency was $20 \mathrm{kHz}$.

\subsection{Experiments}

In order to characterize the band sawing process and construct corresponding stability diagrams, a set of 21 cutting experiments were performed during which the cutting force $\boldsymbol{F}(t)$ and sound 


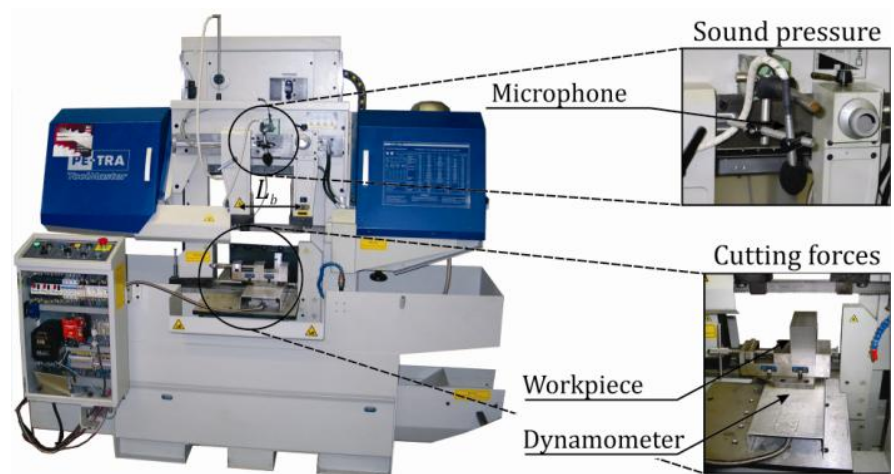

Fig. 1. Double-column horizontal band saw machine used in the experiments and placement of the sensors.

pressure $p(t)$ signals were acquired. The experiments were performed on a full rectangular profile workpiece of width 100 $\mathrm{mm}$ and height $60 \mathrm{~mm}$, made of structural steel (St37 according to DIN 17100). The main process parameters considered in the experiments were the time-dependent cutting speed $v_{c}(t)$, the feed rate $v_{f}$, and the horizontal distance $L_{b}$ between the vertical blade supports. In the cutting experiment the time variation of the cutting speed $v_{c}(t)$ was defined as shown in Fig. 2, with the aim to initiate a spontaneous onset and dying-out of the chatter.

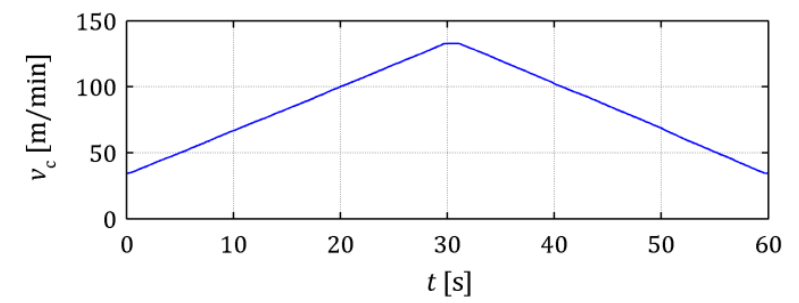

Fig. 2. Time dependent cutting speed profile $v_{c}(t)$.

As evident from Fig. 2, the cutting speed $v_{c}(t)$, was linearly increased over a period of 30 seconds, from $v_{\text {cmin }}=34 \mathrm{~m} / \mathrm{min}$ to the maximum cutting speed $v_{\text {cmax }}=133 \mathrm{~m} / \mathrm{min}$. After reaching and remaining for 2 seconds at the maximum cutting speed $v_{\text {cmax }}$, the cutting speed $v_{c}(t)$ was linearly decreased at the same rate back to the minimum value $v_{c m i n}=34 \mathrm{~m} / \mathrm{min}$. During the experiment with variation of the cutting speed $v_{c}(t)$, the feed rate $v_{f}$ and the distance $L_{b}$ between the blade supports were held constant at a preselected value of $v_{f}=[21,33,45] \mathrm{mm} / \mathrm{min}$, with $L_{b}$ ranging from $250 \mathrm{~mm}$ to $400 \mathrm{~mm}$, with steps of $25 \mathrm{~mm}$. The feed rate $v_{f}$ was chosen such that the forces on the teeth of the cutting blade did not exceed $70 \mathrm{~N}$ per cutting tooth. The experiments were performed at randomly preselected values of $v_{f}$ and $L_{b}$ in order to remove possible bias from the blade wear effect and other system-related effects.

Examples of the sound pressure signals $p(t)$ acquired during the cutting experiment with variation of the cutting speed $v_{c}(t)$ at nine selected different combinations of the feed rate $v_{f}=[21$, $33,45] \mathrm{mm} / \mathrm{min}$ and distance $L_{b}=[250,325,400] \mathrm{mm}$ are shown in Fig. 3. Based on the sound pressure signals $p(t)$ chatter was distinguished from chatter free cutting by the auditory perception of experts and by inspection of the spectrograms obtained by means of short-time power spectra. In relation to this, the presence of chatter is marked in red in Fig. 3. It can be seen that the cutting speed $v_{c}$ and the distance $L_{b}$ have significant effects, whereas the feed rate $v_{f}$ has a smaller effect on process stability and related chatter occurrence.
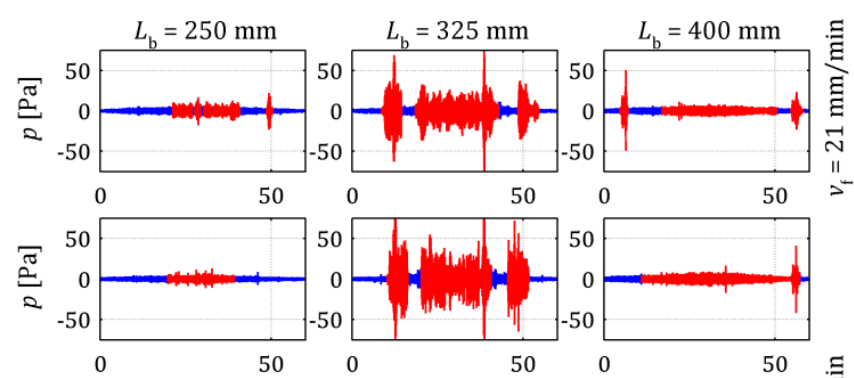

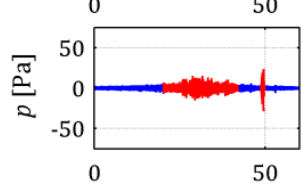

$t$ [s]

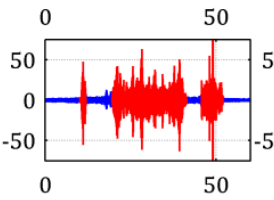

$t$ [s]

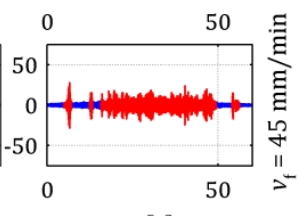

$t[\mathrm{~s}]$
Fig. 3. Sound pressure $p(t)$ signals at three different distances $L_{b}$ and feed rates $v_{f}$.

\section{Theoretical background of recurrence plots analysis}

In this section an advanced method for time series analysis, known as recurrence plots, is presented. The advantage of using recurrence plots (RP) and their quantitative analysis (RQA) is that they are suitable for the analysis of noisy, non-linear and/or non-stationary time series. Recurrent behaviour is an inherent property of oscillating systems and can be mathematically expressed by an $N \times N$ binary matrix with elements [11]

$\boldsymbol{R}_{i j}=\theta\left(\varepsilon-\left\|\boldsymbol{x}_{i}-\boldsymbol{x}_{j}\right\|\right) ; \boldsymbol{x}_{i} \in \mathbb{R}^{m} ; i, j=1, \ldots, N$.

In Eq. (1) $\boldsymbol{x}_{i}$ is the $i$-th state point in the $m$-dimensional state space, $N$ is the number of points in the state space, $\varepsilon$ is a predefined threshold, $\theta(\cdot)$ is the Heaviside function and $\|\cdot\|$ is some chosen, in our case Euclidian norm. The value of the $\boldsymbol{R}_{i j}$ is either 1 or 0 , depending on the distance between $\boldsymbol{x}_{i}$ and $\boldsymbol{x}_{j}$ and the selected threshold $\varepsilon$. Graphical presentation of the recurrence matrix $\boldsymbol{R}_{i j}$, with "ones" shown in colour and "zeros" shown in white, is known as a recurrence plot. In order to reconstruct the state space from a time series, the embedding theorem [13] can be employed where the states are determined by the delay vectors

$\boldsymbol{x}_{i}=\left(x_{i}, x_{i-\tau}, \ldots, x_{i-(m-1) \tau}\right)$

where $x_{i}=x(i \Delta t)$ is a scalar time series with a sampling time $\Delta t$, $\tau$ is the time delay, and $m$ is the embedding dimension. Assuming that the time series originates from a deterministic dynamic system and is stationary, then for a large enough embedding dimension $m$ the theorem states that the reconstructed state space is bound to have the same dynamic properties as that formed by the original variables of the observed system [13]. Whereas these restrictions are crucial to methods of non-linear time series analysis [14], they are not so vital with regard to recurrence plots. With respect to the latter, the problem of finding proper embedding dimension may be an elusive one, so that an embedding dimension of $m=10$ is routinely used [15], [16]. Besides the embedding parameters $\tau$ and $m$ it is important to carefully choose the threshold $\varepsilon$. For this purpose various options have been proposed and are summarized in [17], but it is vital that a common criterion is chosen when comparing different time series. When analysing non-stationary data it is instructive to choose $\varepsilon$ such that the recurrence rate is equal for all the compared recurrence plots. Additionally, when a windowed RQA is performed at least $1 \%$ of the recurrence rate needs to be assured in every segment (window) of the time series [18]. The 

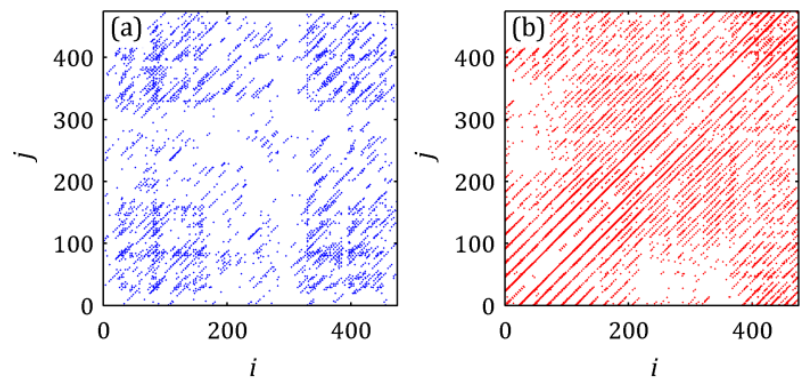

Fig. 4. Recurrence plots: (a) Chatter free cutting, and (b) Chatter cutting

recurrence plots generated from the sound pressure signals $p(t)$ of chatter free and chatter cutting are shown in Fig. 4.

For the analysis of the stability of the band sawing process, sound pressure signals $p(t)$ were partitioned into window segments of $t_{w}=50 \mathrm{~ms}$. The recurrence plot for each segment was constructed according to Eq. 1, with a time delay of $\tau=3$, which was determined from mutual information [14], an embedding dimension $m=10$ and a threshold $\varepsilon=2.3 \sigma$, where $\sigma$ is the standard deviation of the measured time series amplitude. The texture of the recurrence plot can be characterized statistically, based on the diagonal and vertical line lengths of the recurrence plot [17]. The diagonal characteristics of the recurrence plot are based on the histogram $P(l)$ of the diagonal lines of length $l$

$P(l)=\sum_{i, j=1}^{N}\left(1-\boldsymbol{R}_{i-1, j-1}\right)\left(1-\boldsymbol{R}_{i+l, j+l}\right) \prod_{k=0}^{l-1} \boldsymbol{R}_{i+k, j+k}$.

In our case of stability analysis the determinism DET will be used, which is defined as the ratio of the recurrence points that form diagonal structures to all recurrence points

$D E T=\frac{\sum_{l=l_{\min }}^{N} l P(l)}{\sum_{l=1}^{N} l P(l)}$.

Regular deterministic-like time series form long diagonals with very few isolated recurrence points, so the determinism is close to one, whereas in the case of a stochastic time series it is close to zero.

\section{Results}

\subsection{Stability diagrams}

The determinism DET was calculated from the sound pressure signals $p(t)$ at the selected feed rates of $v_{f}(t)=[21,33,45]$ $\mathrm{mm} / \mathrm{min}$. The distance between the blade supports $L_{b}$ varied between 250 and $450 \mathrm{~mm}$, with increments of $25 \mathrm{~mm}$. The cutting speed $v_{c}$ was varied in the triangular manner (Fig. 2) as described in section 2. A mowing window time segment $t_{w}$ used in the calculation of determinism DET was set to $t_{w}=0.050 \mathrm{~s}$.

The result of the analysis consist of a scalar field of the determinism DET as it depends on the cutting speed $v_{c}$, the distance between the blade supports $L_{b}$, and the feed rate $v_{f}$, i.e. $\operatorname{DET}\left(v_{c}, L_{b}, v_{f}\right)$ as shown in Fig. 5. The scalar field corresponds to the stability diagram of the structural steel St37 profile band sawing, where the values of the $D E T$ close (red) denote chatter regions and the values close to zero (blue) denote regular chatterfree cutting. The left half of each of the diagrams represents the $D E T$ for the increasing and the right half for the decreasing cutting speed $v_{c}$, according to the $v_{c}(t)$ profile shown in Fig. 2. It can be seen that the chatter regions in the stability diagrams are not connected together but rather form isolated areas of alternating chatter - chatter free cutting regions. A slight
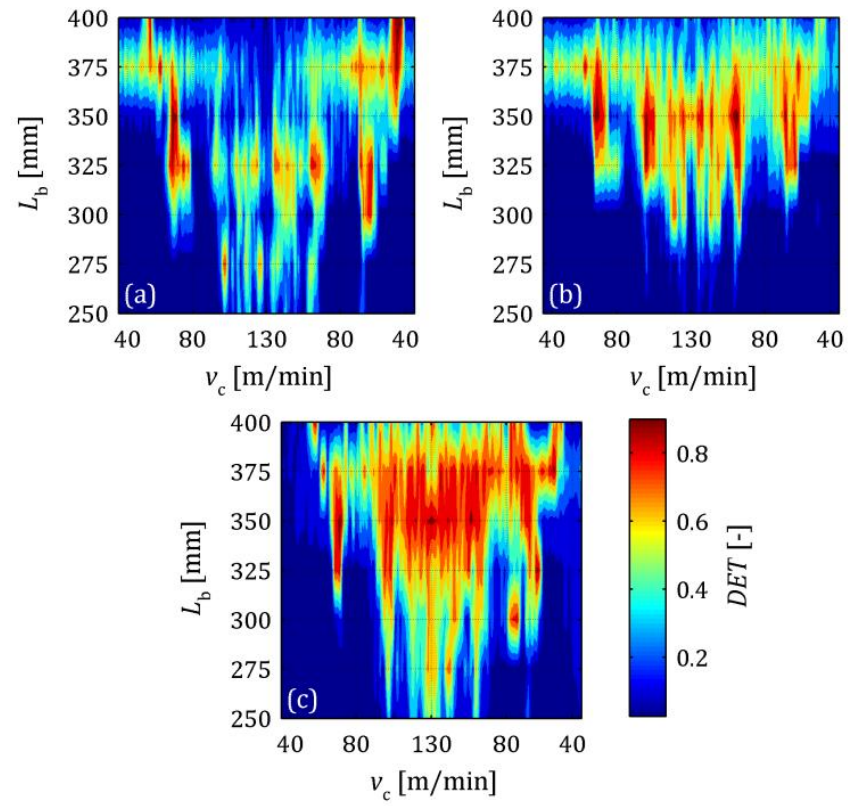

Fig. 5. Stability diagrams defined by the values of $\operatorname{DET}\left(v_{c}, L_{b}, v_{f}\right)$ in the process parameter space of the cutting speed $v_{c}$, the distance $L_{b}$ for the feed rates $v_{f}(t)=[21,33,45] \mathrm{mm} / \mathrm{min}$. (a) Feed rate $v_{f}(t)=21 \mathrm{~mm} / \mathrm{min}$. (b) Feed rate $v_{f}(t)=33 \mathrm{~mm} / \mathrm{min}$. (c) Feed rate $v_{f}(t)=45 \mathrm{~mm} / \mathrm{min}$.

asymmetry of the stability diagrams can also be observed with respect to the increasing and decreasing cutting speed $v_{c}(t)$, indicating the presence of a hysteresis, which is characteristic for the chatter onset instability. More in particular the chatter onset cutting speed $v_{c}$ is slightly higher than the chatter dying out $v_{c}$. The distance $L_{b}$ between the blade supports directly affects the first chatter onset and the last chatter dying-out cutting speed $v_{c}$ and the corresponding width of the chatter region, in the space of the cutting speed $v_{c}$, which nonlinearly increases with an increase in the distance $L_{b}$. The feed rate $v_{f}$ has less effect on the width of the chatter region, but is does affect the overall proportion of the chatter to chatter free cutting regions, which increases linearly with an increasing feed rate $v_{f}$.

Based on the presented results, the most influential parameters are the cutting speed $v_{c}(t)$ and the distance $L_{b}$. However, the distance between the blade supports is determined by the width of the workpiece, and since it is not an easily adjustable parameter it therefore cannot be used as the chatter control parameter. On the other hand the chatter onset characterized by the $D E T$ is non-linearly and non-monotonically dependent on the cutting speed $v_{c}$, the variation of which is easily performed. The latter infers the possibility of cutting at low cutting speed $v_{c}$ below or at high cutting speed $v_{c}$ above the chatter region, showing that the cutting speed $v_{c}$ can be used as an effective control parameter for chatter avoidance.

\subsection{Chatter avoidance}

The highly non-linear and non-monotonic dependence of the $D E T$ on the cutting speed $v_{c}$ observed from the stability diagrams shown in Fig. 5 means that two different chatter avoidance strategies are possible:

1. a decrease in the cutting speed $v_{c}$ below the isolated chatter region, or

2. an increase in the cutting speed $v_{c}$ above the isolated chatter region.

In order to experimentally verify these two chatter avoidance strategies, an additional cutting experiment was designed based 


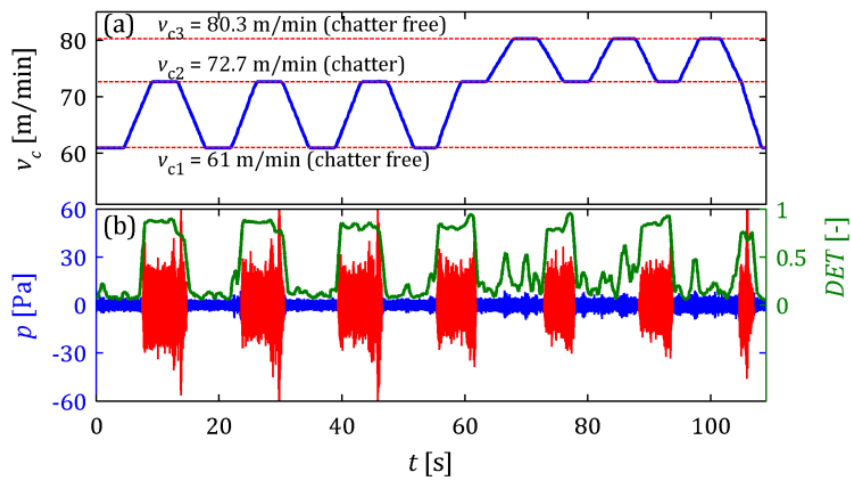

Fig. 6. Chatter avoidance experiment: (a) the cutting speed $v_{c}(t)$ variation and (b) the corresponding sound pressure $p(t)$ and determinism DET.

on the stability diagram at the feed rate $v_{f}(t)=33 \mathrm{~mm} / \mathrm{min}$. From the stability diagram (Fig. $5 \mathrm{~b}$ ), at a distance between the blade supports of $L_{b}=350 \mathrm{~mm}$, cutting speeds of $v_{c 1}=61.0$, $v_{c 2}=72.7$ and $v_{c 3}=80.3 \mathrm{~m} / \mathrm{min}$ were selected, which corresponded to chatter free, chatter, and chatter free cutting. In order to experimentally simulate chatter avoidance with a decreasing and increasing cutting speed $v_{c}$, the cutting speed $v_{c}(t)$ was varied as is shown in Fig 6a. In Fig. 6b the resulting fluctuations of the sound pressure $p(t)$ with marked regions of chatter in red colour, and the values of the corresponding determinism $D E T$ are shown. It can be seen that, in the case of linear variation of the cutting speed $v_{c}$ from $v_{c 1}$ to $v_{c 2}$ and back to $v_{c 1}$, chatter avoidance is achieved by a decrease in $v_{c}$ from $v_{c 2}$ to $v_{c 1}$, which is evident in the decreased variance of the acquired sound pressure $p(t)$ and clearly indicated by values of $D E T$ close to zero. Contrary to this, in the case of the linear variation of $v_{c}$ from $v_{c 2}$ to $v_{c 3}$ and back to $v_{c 2}$ chatter avoidance is achieved by an increase in $v_{c}$ from $v_{c 2}$ to $v_{c 3}$ which is again reflected in the lower variance of the $p(t)$ and values of $D E T$ close to zero, characteristic for a chatter free cutting regime.

As inferred in [19], the absolute cutting forces $|\boldsymbol{F}|$ according to the theoretical predictions are inversely proportionate to the cutting speed $v_{c}$. Indeed, the mean absolute cutting force values estimated from the cutting force $\boldsymbol{F}(t)$ measurements at $v_{c 1}$ and $v_{c 3}$ were $|\boldsymbol{F}|=360 \mathrm{~N}$ and $|\boldsymbol{F}|=301 \mathrm{~N}$, respectively. This indicates that, although blade wear in band sawing is expected to increase at a higher cutting speed $v_{c}[20]$, the time of cut, which contributes significantly to the cutting economy, can be lowered by increasing both the feed rate $v_{f}$ and the cutting speed $v_{c}$. It is thus advisable to avoid chatter in band sawing by increasing the cutting speed.

\section{Conclusions}

The paper presents a method of stability analysis of the band sawing process on a horizontal band sawing machine. For the construction of stability diagrams the recurrence plot characteristic, i.e. the determinism DET, was employed. The process stability was analysed within the process parameter space, defined by the cutting speed $v_{c}$., the feed rate $v_{f}$, and the distance between the cutting blade supports $L_{b}$. It was shown that the proposed method for the construction of stability diagrams yields $D E T$ values which are close to one during chatter, and close to zero in the case of regular, chatter-free cutting.

The results show that the cutting speed is the most influential process parameter affecting the process stability. The chatter onset, characterized by the determinism $D E T$, is non-linearly and non-monotonically dependent on the cutting speed. In this connection, it was observed that the distance $L_{b}$ between the blade supports directly affects the first chatter onset and the corresponding dying-out cutting speed, i.e. the width of the chatter region in the space of the cutting speed, which increases nonlinearly with increases in the distance $L_{b}$. On the other hand, the feed rate $v_{f}$ has less effect on the width of the chatter region in the cutting speed parameter space. However, it affects the overall proportion of the chatter-to-chatter free cutting regions, which linearly increases with increasing values of the feed rate $v_{f}$.

The observed non-monotonic nature of the chatter regions indicated by the $D E T$ in the process parameter space $\left(v_{c}, L_{b}\right.$. $\left.v_{f}\right)$, and ease of variation of the cutting speed $v_{c}(t)$ suggests that chatter can be avoided either by reducing or by increase the cutting speed $v_{c}$. However, when the cutting speed $v_{c}$ is increased, the decrease in the cutting forces and the cutting time are related. Thus, in order to improve the economy of band sawing it is advisable to avoid chatter by increasing the cutting speed $v_{c}$.

\section{Acknowledgments}

This work was supported by ARRS - the Slovenian Research Agency, Research program P02-241-Synergetics of complex systems and processes.

\section{References}

[1] Altintas, Z., Weck, M., 2004, ChatterStability of Metal Cutting and Grinding, Annals of the CIRP, 53/2: 619-642.

[2] Alspaugh, D.W., 1967, Theoretical and Experimental Band Saw Vibrations, Journal of the Franklin Institute, 283/4: 328-338.

[3] Delio, T., Tlusty, I., 1992, Use of audio signals for chatter detection and control, Journal of Engineering for Industry, 114/2:146-157.

[4] Rahman, M., 1986, Detection of the onset of chatter vibration, Journal of Sound and Vibration, 109/2:193-205.

[5] Tangjitsitcharoen, S., Moriwaki, T., 2008, Intelligent monitoring and identification of cutting states of chips and chatter on CNC turning machine, Journal of Manufacturing Processes, 10: 40-46.

[6] Tangjitsitcharoen, S., 2009, In-process monitoring and detection of chip formation and chatter for CNC turning, Journal of Materials Processing Technology, 209:4682-4688.

[7] Thaler, T., Potočnik, P., Bric, I., Govekar, E., 2014, Chatter detection in band sawing based on discriminant analysis of sound features, Applied Acoustics, 77: 114121.

[8] Cao, H., Lei, Y., He, Z., 2013, Chatter identification in end milling process using wavelet packets and Hilbert-Huang transform, International Journal of Machine Tools \& Manufacture, 69: 11-19.

[9] Eckmann, J.-P., Kamphorst, S.O., Ruelle, D., 1987, Recurrence Plots of Dynamical Systems, Europhysics Letters, 5:973-977.

[10] Marwan, N., Wessel, N., Meyerfeldt, U., Schirdewan, A., Kürths, J., 2002, Recurrence-plot-based measures of complexity and their application to heart-ratevariability data, Physical Review E, 66:026702-1-2-8.

[11] Litak, G., Sen, A.K. Syta, A., 2009, Intermittent and chaotic vibrations in a regenerative cutting process, Chaos Solitons \& Fractals, 41/4: 2115-2122.

[12] Rajesh, V.G., Namboothiri, V.N.N., 2010, Utilizing Recurrence Quantification Analysis for Chatter Detection in Turning, Proceedings of the International Manufacturing Science and Engineering Conference - Advances in Modelling, Analysis, and Simulation of Manufacturing Processes 2:149-156.

[13] Takens, F., 1980, Detecting strange attractors in turbulence, Dynamical Systems and Turbulence, Proceedings of a Symposium Held at the University of Warwick, 366-381.

[14] Kantz, H., Schreiber, T., 1997, Nonlinear time series analysis, Cambridge University Press, 1997.

[15] Gao, J., Cai, H., 2000, On the structures and quantification of recurrence plots, Physics Letters A, 270: 75-87.

[16] Zbilut, J.P. Giuliani, A., Webber Jr., C.L., 1998, Recurrence quantification analysis and principal components in the detection of short complex signals, Physics Letters A, 237:131-135.

[17] Marwan, N., Romano, M.C., Thiel, M., Kürths, J., 2007, Recurrence plots for the analysis of complex systems, Physics Reports, 438:237-329.

[18] Zbilut, J.P. Giuliani, A. Webber Jr., C.L., 2002, Recurrence quantification based Liapunov exponents for monitoring divergence in experimental data, Physics Letters A, 297: 173-181.

[19] Sarwar, M., Persson, M., Hellbergh, H., Haider, J., 2009, Measurement of specific cutting energy for evaluating the efficiency of band sawing different workpiece materials, International Journal of Machine Tools \& Manufacture, 49: 958-965.

[20] Saglam, H., 2011, Tool wear monitoring in band sawing using neural networks and Taguchis design of experiments, International Journal of Advanced Manufacturing Technology, 55:969-982. 